\title{
ON THE EXCESS CHARGE OF A RELATIVISTIC STATISTICAL MODEL OF MOLECULES WITH AN INHOMOGENEITY CORRECTION
}

\author{
HONGSHUO CHEN \\ College of Mathematics and Statistics, Chongqing University, Chongqing 401331, \\ China
}

HEINZ SIEDENTOP

Mathematisches Institut

Ludwig-Maximilans-Universität München, Theresienstraße 39

80333 München

Germany

and Munich Center for Quantum Science and Technology (MCQST)

Schellingstr. 4

80799 München, Germany

\begin{abstract}
We show that the molecular relativistic Thomas-Fermi-Weizsäcker functional consisting of atoms of atomic numbers $Z_{1}, \ldots, Z_{k}$ has a minimizer, if the particle number $N$ is constrained to a number less or equal to the total nuclear charge $Z:=Z_{1}+\ldots+Z_{K}$. Moreover, there is no minimizer, if the particle number exceeds $2.56 Z$. This gives lower and upper bounds on the maximal ionization of heavy atoms.
\end{abstract}

\section{INTRODUCTION}

Shortly after the advent of quantum mechanics it became clear that the many particle problem of interacting quantum systems cannot be solved exactly much like in classical quantum mechanics. Thomas [26 and Fermi [9, 10] developed a density functional theory that turned out to describe atoms of large atomic numbers $Z$ asymptotically correct as far as the energy and the density on the scale $Z^{-1 / 3}$ is concerned (Lieb and Simon [22]). Thomas and Fermi assumed in their intuitive derivation that the potential would be locally constant. Their functional (Lenz [18) generalized to molecules with atomic nuclei of atomic number $Z_{k}$ situated at $R_{k}$ reads

(1) $\mathcal{E}^{\mathrm{nTF}}(\rho):=\int_{\mathbb{R}^{3}} \mathrm{~d} x\left(\frac{3}{10} \gamma \rho^{\frac{5}{3}}(x)-\sum_{k=1}^{K} \frac{Z_{k} \rho(x)}{\left|x-R_{k}\right|}\right)+D[\rho]+\sum_{1 \leq k<l \leq K} \frac{Z_{k} Z_{l}}{\left|R_{k}-R_{l}\right|}$

where $D[\rho]:=D(\rho, \rho)$ is the quadratic form associated with the Hermitian form

$$
D(\rho, \sigma):=\frac{1}{2} \iint_{\mathbb{R}^{3} \times \mathbb{R}^{3}} \mathrm{~d} x \mathrm{~d} y \frac{\overline{\rho(x)} \sigma(y)}{|x-y|} .
$$

E-mail addresses: hongshuo.chen@gmail.com, h.s@lmu.de.

Date: July 3, 2020. 
The functional is naturally defined on all nonnegative $\rho \in L^{5 / 3}\left(\mathbb{R}^{3}\right)$ with finite Coulomb energy $D[\rho]$. The positive constant $\gamma$ is physically $\left(3 \pi^{2}\right)^{2 / 3}$. In the following we will write $Z$ for the sum of the nuclear charges, i.e.,

$$
Z:=Z_{1}+\ldots+Z_{K}
$$

By Teller's lemma (Teller [25, see also Simon [23, Section III.9]) the infimum of this functional taken over the densities $\rho$ in the above set and all pairwise different nuclear positions $R_{1}, \ldots, R_{K} \in \mathbb{R}^{3}$ is the sum of the infima of the atomic functional (no binding) and scales in $\gamma$ and $Z_{k}$ :

$$
\mathcal{E}^{\mathrm{nTF}}(\rho) \geq-\gamma^{-1} \underbrace{e_{\mathrm{TF}} \sum_{k=1}^{K} Z_{k}^{7 / 3}}_{A:=}
$$

where $-e_{\mathrm{TF}}$ is the infimum of the Thomas-Fermi functional with $K=1, Z=Z_{1}=$ 1 , and $\gamma=1$.

Later Weizsäcker added a correction accounting for rapidly changing potentials. The resulting functional of the density, the nonrelativistic TFW-functional, for atom with $q$ spin states per electron is

$$
\int_{\mathbb{R}^{3}} \mathrm{~d} x \frac{1}{2}|\nabla \sqrt{\rho(x)}|^{2}+\mathcal{E}^{\mathrm{nTF}}(\rho) .
$$

Note that $1 / 2$ in front of the gradient term is the original constant used by Weizsäcker. However, there are also other constants discussed, e.g., 1/18 emanating from the gradient expansion (Kirzhnits [17, Hodges [16]), or 1/10 adapting the Scott correction of the TFW functional to its physical value (Yonei and Tomishima [27, see also Lieb and Liberman 20] for a slightly different numerical value).

This so called inhomogeneity correction yields an exponential decay of the atomic density as opposed the power decay of TF-theory and makes the potential finite at the nucleus as opposed to the pure Thomas-Fermi case which has a $|x|^{-3 / 2}$ singularity at the nucleus. Also the excess charge $Q:=N-Z$, where $N$ is the maximal number of electrons that the atom can bind, is raised from 0 to a positive number which Benguria and Lieb [4] bound by a constant of order one. This reflects the experimental fact, that for real atoms there are no doubly charged negative atomic ions.

From the physical point of view, however, the description of large atoms with nonrelativistic theories is of limited interest, since large atomic numbers result in velocities of the innermost electrons that require a relativistic description. This has well known consequence to the quantum energy (Solovej et al 24, Frank et al [12, 13, and Handrek and Siedentop [15]) and for the density (Frank et al [11]).

A generalization to relativistic density functionals, however, suffered from the fact that the naive generalization leads to an energy functional that is unbounded from below and yields a relativistic TF-equation whose solution has necessarily infinitely many particles because of a $|x|^{-3}$ singularity at the origin. (For a review see Gombas [14, §14].)

Dreizler and Engel [7] offered a solution to this problem: they derived a relativistic functional from quantum electrodynamics and later solved it numerically for atoms (Dreizler and Engel [8]). Following Engel and Dreizler we write it in terms of the Fermi momentum $p$ given by

$$
p(x):=\left(3 \pi^{2} \rho(x)\right)^{1 / 3},
$$

instead of the density $\rho$. The TFW part (dropping the Dirac term and and an overall trivial factor of $m c^{2}$ ) reads in the case of molecules consisting of $K$ atoms 
of atomic numbers $Z_{1}, \ldots, Z_{K}$ located at positions $R_{1}, \ldots, R_{K}$

(6) $\mathcal{E}^{\mathrm{TFW}}(p)$

$$
:=\mathcal{T}^{\mathrm{W}}(p)+\mathcal{T}^{\mathrm{TF}}(p)-\sum_{k=1}^{K} \frac{\alpha_{S} Z_{k}}{3 \pi^{2}} \int_{\mathbb{R}^{3}} \mathrm{~d} x \frac{p^{3}(x)}{\left|x-R_{k}\right|}+\frac{\alpha_{S}}{9 \pi^{4}} D\left[p^{3}\right]+\sum_{1 \leq k<l \leq K} \frac{\alpha_{S} Z_{k} Z_{l}}{\left|R_{k}-R_{l}\right|}
$$

where $\alpha_{S}$ is the Sommerfeld fine structure constant, which is $1 / c$ in Hartree units and has the physical value of about $1 / 137$. (The atomic case is $K=1$ in which case we can assume $R_{1}=0$. Of course, then $Z_{1}=Z$.) The Thomas-Fermi part of the kinetic energy is

$$
\mathcal{T}^{\mathrm{TF}}(p):=\frac{1}{8 \pi^{2}} \int_{\mathbb{R}^{3}} \mathrm{~d} x t^{\mathrm{TF}}(p(x))
$$

with

$$
t^{\mathrm{TF}}(s):=s\left(s^{2}+1\right)^{3 / 2}+s^{3}\left(s^{2}+1\right)^{1 / 2}-\operatorname{arsinh}(s)-\frac{8}{3} s^{3} .
$$

The Weizsäcker part of the kinetic energy is

$$
\mathcal{T}^{\mathrm{W}}(p):=\frac{3 \lambda}{8 \pi^{2}} \int_{\mathbb{R}^{3}} \mathrm{~d} x(\nabla p(x))^{2} f(p(x))^{2}
$$

with

$$
f(t):=\sqrt{\frac{t}{\sqrt{t^{2}+1}}+2 \frac{t^{2}}{t^{2}+1} \operatorname{arsinh}(t)} .
$$

The constant $\lambda$ is positive.

We note that the ultrarelativistic limit of this functional - dropping the regularizing arsinh in the Weizsäcker term - has been considered before by Benguria et al [5]: it turns out that stability of matter holds for small enough $Z$, whereas the functional is unbounded from below, if the $Z$ is large. A preliminary investigation of the massive functional appeared in 6 .

In this paper we consider the massive case. As opposed to the above case and many other relativistic models, we will show that $\mathcal{E}^{\mathrm{TFW}}$ is bounded from below for all $Z_{1}, \ldots, Z_{K}, R_{1}, \ldots, R_{k}$ and $N$, in fact, we will show that the bound can be uniform in $R_{1}, \ldots, R_{K}$ and $N$. This will be achieved by showing an upper bound on the number of particles that can be bounded in terms of the nuclear charge $Z$.

Next we specify the space of allowed Fermi momenta $p$. To this end we introduce the antiderivative

$$
F(t):=\int_{0}^{t} \mathrm{~d} s f(s)
$$

We define

$$
P:=\left\{p \in L^{4}\left(\mathbb{R}^{3}\right) \mid p \geq 0, D\left[p^{3}\right]<\infty, F \circ p \in D^{1}\left(\mathbb{R}^{3}\right)\right\}
$$

where $D^{1}\left(\mathbb{R}^{3}\right)$ is the space of all locally integrable functions on $\mathbb{R}^{3}$ which decay at infinity and have a square integrable gradient (Lieb and Loss [21, Section 8.2]). For later purposes we also introduce for $N \geq 0$

$$
P_{N}:=\left\{\rho \mid \sqrt[3]{\rho} \in P, \int_{\mathbb{R}^{3}} \mathrm{~d} x \rho(x) \leq N\right\} .
$$

It turns out that all terms occurring in the TFW functional are well defined on $P_{N}$ as we shall see in the proof of Theorem 1, In particular the Weizsäcker term becomes simply

$$
\tilde{T}^{\mathrm{W}}(F \circ p):=\int_{\mathbb{R}^{3}} \mathrm{~d} x|\nabla(F \circ p)|^{2}(x) .
$$


For the proof on the excess charge, it will be convenient to write the energy functional in terms of $\chi:=F \circ p$.

Our first results are:

Theorem 1 (Stability). For any $Z_{\infty}>0$, and any $K \in \mathbb{N}$ there exists a constant $c\left(Z_{\infty}^{7 / 3} \cdot K\right)$ depending only on $Z_{\infty}^{7 / 3} \cdot K$ such for all $N \in \mathbb{R}_{+}, Z_{1}, \ldots, Z_{K} \in\left[0, Z_{\infty}\right]$, and pairwise different $R_{1}, \ldots, R_{k} \in \mathbb{R}^{3}$

$$
\inf \mathcal{E}^{\mathrm{TFW}}\left(P_{N}\right) \geq-N-c\left(Z_{\infty}^{7 / 3} \cdot K\right)
$$

Note the surprising fact that - unlike in many other relativistic models of Coulomb systems - there is no critical nuclear charge beyond which the energy is unbounded from below. As the proof will show this is due to the occurrence of the arsinh in the Weizsäcker term making it logarithmically stronger than the Coulomb singularity. - In Engel and Dreizler's formal derivation of the functional from quantum electrodynamics this occurrence is a consequence of the necessary renormalization.

Furthermore, we remark that - unlike the case treated here - a lower bound which is linear in $N$ and even in $K$ - of the nonrelativistic TFW-functional is obvious, since it is bounded from below by the nonrelativistic TF-functional. The stability of the TF-functional follows then by Teller's no-binding theorem and the fact that the excess charge of atoms is zero. The same is true here. However, the relativistic TF kinetic energy is not strong enough to prevent collapse: its infimum is $-\infty$ (Gombas [14, Chapter III, §14]).

Theorem 2 (Existence of Minimizers). For any $N, Z_{1}, \ldots, Z_{K} \geq 0$, the functional $\mathcal{E}^{\mathrm{TFW}}$ has a minimizer $p_{N}$ on $P_{N}$, and, moreover, if $p_{N}$ is a minimizer on $P_{N}$ for $N \leq Z$, then its particle number $\int_{\mathbb{R}^{3}} \mathrm{~d} x p_{N}(x)^{3} /\left(3 \pi^{2}\right)$ is equal to $N$, i.e., the minimizer occurs on the boundary of $P_{N}$.

Note that we do not claim uniqueness of the minimizer: unlike the nonrelativistic TFW functional, the relativistic Weizsäcker correction is not a convex functional of the density $\rho$, i.e., the standard tool for showing uniqueness of minimizers is not available.

To formulate the next theorem we introduce the function $H$ on the positive real line by

$$
H(s):=F(s) /\left(s F^{\prime}(s)\right)
$$

and write for its minimum and maximum

$$
a:=\inf H\left(\mathbb{R}_{+}\right) \text {and } b:=\sup H\left(\mathbb{R}_{+}\right) .
$$

Numerically $a=0.6116832747$.

Theorem 3 (Bound on the Excess Charge). For all $N \in \mathbb{R}_{+}$, all $K \in \mathbb{N}$, all $Z_{1}, \ldots, Z_{K} \in \mathbb{R}_{+}$, and all pairwise different $R_{1}, \ldots, R_{K} \in \mathbb{R}^{3}$ the minimizer $\rho$ of $\mathcal{E}^{\mathrm{TFW}}$ on $P_{N}$ fulfills

$$
\int_{\mathbb{R}^{3}} \mathrm{~d} x \rho(x)<\frac{2}{\sqrt{a}} Z
$$

Note that inserting the numerical value of $a$ gives

$$
\int_{\mathbb{R}^{3}} \mathrm{~d} x \rho(x)<2.56 Z
$$

This result shows in particular that the relativistic TFW functional cannot bind infinitely many electrons. Of course one should - presumably - regard this only as a first step, since one might conjecture also here that the maximal excess charge $Q$ is - like in the nonrelativistic case - bounded by one [4]. 
Finally, we note the following immediate consequence of Theorems 1 and 3 By Theorem $3 \inf _{N \in \mathbb{R}_{+}} \inf \mathcal{E}^{\mathrm{TFW}}\left(P_{N}\right) \geq \inf \mathcal{E}^{\mathrm{TFW}}\left(P_{2 a^{-1 / 2} Z}\right)$. Thus, when minimizing $\mathcal{E}^{\mathrm{TFW}}$, we might, right from the beginning, restrict to the case that $N \leq 2 a^{-1 / 2} Z$. Moreover, $Z=Z_{1}+\ldots+Z_{K} \leq Z_{\infty} K$. Inserting this in (19) gives the following corollary:

Corollary 1. For any $Z_{\infty} \geq 1$, and any $K \in \mathbb{N}$ there exists a constant $d\left(Z_{\infty}^{7 / 3} \cdot K\right)$ such for all $N \in \mathbb{R}_{+}, Z_{1}, \ldots, Z_{K} \in\left[0, Z_{\infty}\right]$, and pairwise different $R_{1}, \ldots, R_{k} \in \mathbb{R}^{3}$

$$
\inf \mathcal{E}^{\mathrm{TFW}}\left(P_{N}\right) \geq-d\left(Z_{\infty}^{7 / 3} \cdot K\right) .
$$

Note that physics suggests that $Z_{\infty}$ can be chosen uniformly in $K$, since there are no elements known with a nuclear charge higher than 119 , i.e., $Z_{\infty}=119$ is a reasonable assumption. Assuming such a uniform bound on $Z_{1}, \ldots, Z_{K}$ yields a lower bound on the energy which depends only on the number of nuclei which are present in the Coulomb system at hand.

The structure of the remaining part is basically structured according to our three main results: In Section 2 we prove the stability result Theorem 1 The main part is a detailed lower bound on the energy. The essential input is an estimate on the potential energies using the nonrelativistic TF-theory and an involved estimate of the resulting negative nonrelativistic TF kinetic energy in terms of relativistic Weizsäcker term and the massless relativistic TF kinetic energy. Section 3 contains the existence result which is inspired by Benguria et al [2]. Section 4 and 5$]$ show the bounds on the ionization. Although the upper bound is inspired by Benguria's early unpublished proof of the bound $N<2 Z$ for the excess charge of the ThomasFermi model, namely to integrate the Euler equation against a suitable weight. (See also the application of this idea by Lieb [19] for the Schrödinger equation and Benguria et al 3 for the Hellmann-Weizsäcker functional.) However, it cannot be applied in a straight forward manner in the present context. We need to transform the functional and estimate the resulting functional using some estimates on the function $F$ of (11) and its inverse. We are able to control the errors so that we loose only slightly compared to the above mentioned nonrelativistic results [19, 3 .

Finally, we collect some basic facts needed throughout the proves in the Appendix: Appendix $\mathrm{A}$ gives some results that are a consequence of the phase space representation of the kinetic energy of the relativistic TF-functional. Appendix B gives the needed basic properties of $F$.

\section{Proof of Stability}

In this section we will show that the infimum of the energy functional is bounded from below by a bound that is linear in the number of involved electrons $N$ plus a constant depending on the number of nuclei $K$ and the maximal occurring atomic number $Z_{\infty}$.

We begin with the definition of two cut-off radii $R$ and $\tilde{R}$ depending on parameters $\alpha$ and $\beta$. These are defined as minimizers of two functions $F_{\alpha}$ and $\tilde{F}_{\alpha, \beta}$

Definition 1. We define the function $R$ :

$$
\begin{aligned}
R: \mathbb{R}_{+} & \rightarrow \mathbb{R}_{+} \\
\beta & \mapsto R_{\beta}
\end{aligned}
$$

where, given $\beta \in \mathbb{R}_{+}, R_{\beta}$ is the unique minimizer of $F_{\beta}$ given by

$$
F_{\beta}(r):=\frac{1}{r \operatorname{arsinh}(r)^{3}}+\frac{r}{\beta}
$$

in the variable $r \in \mathbb{R}_{+}$. 
Furthermore, we define the function $\tilde{R}$ :

$$
\begin{aligned}
\tilde{R}: \mathbb{R}_{+}^{2} & \rightarrow \mathbb{R}_{+} \\
(\alpha, \beta) & \mapsto \tilde{R}_{\alpha, \beta}
\end{aligned}
$$

where, given $(\alpha, \beta) \in \mathbb{R}_{+}^{2}, \tilde{R}_{\alpha, \beta}$ is the unique minimizer of

$$
\tilde{F}_{\alpha, \beta}(r):= \begin{cases}\frac{1}{r \operatorname{arsinh}\left(R_{\beta}\right)^{3}}+\frac{r}{\alpha} & r \geq R_{\beta} \\ \frac{1}{r^{4}}\left(\frac{R_{\beta}}{\operatorname{arsinh}\left(R_{\beta}\right)}\right)^{3}+\frac{r}{\alpha} & r<R_{\beta}\end{cases}
$$

in the variable $r \in \mathbb{R}_{+}$.

That these definitions are meaningful is a consequence of Lemma 1 below: Because the function $F_{\beta}$ with $\beta \in \mathbb{R}_{+}$fixed, is continuous and diverges to $+\infty$ for $R \rightarrow 0$ or $R \rightarrow \infty$, its infimum on $\mathbb{R}_{+}$is attained. Because of the strict convexity of $F_{\beta}$ its minimizer $R_{\beta}$ is uniquely defined. Thus $R$ is well defined.

Since $R_{\beta}$ is well defined, it follows that $\tilde{F}_{\alpha, \beta}$ is well defined. Now, we repeat the above argument to define $\tilde{R}_{\alpha, \beta}$ again using Lemma 1 .

Lemma 1. The functions $F_{\beta}$ and $\tilde{F}_{\alpha, \beta}$ are continuous, diverge to $+\infty$ at 0 and $\infty$, and are strictly convex.

Proof. Obviously $F_{\beta}$ is continuous and has the stated behavior at 0 and $\infty$. That it is strictly convex follows from the fact that it is twice differentiable (even real analytic) on $\mathbb{R}_{+}$and the second derivative is positive, since its first derivative

$$
F_{\beta}^{\prime}(r)=-\frac{1}{r^{2} \operatorname{arsinh}(r)^{3}}-\frac{3}{r \operatorname{arsinh}(r)^{4} \sqrt{1+r^{2}}}+\frac{1}{\beta}
$$

is obviously strictly increasing.

The functions $\tilde{F}_{\alpha, \beta}$ are, by inspection, also continuous and diverge to $\infty$ at 0 and $\infty$. Outside $r=R_{\beta}$ they are also twice differentiable (in fact again real analytic) and have the derivative

$$
\tilde{F}_{\alpha, \beta}^{\prime}(r)=\left\{\begin{array}{ll}
-\frac{1}{r^{2} \operatorname{arsinh}\left(R_{\beta}\right)^{3}}+\frac{1}{\alpha} & r>R_{\beta} \\
-\frac{4}{r^{5}}\left(\frac{R_{\beta}}{\operatorname{arsinh}\left(R_{\beta}\right)}\right)^{3}+\frac{1}{\alpha} & r<R_{\beta}
\end{array} .\right.
$$

Outside $R_{\beta}$ these functions are monotone increasing. In addition at $R_{\beta}$ we have a jump of positive height, namely

$$
\lim _{r \nearrow R_{\beta}} \tilde{F}_{\alpha, \beta}^{\prime}(r)-\lim _{r \searrow R_{\beta}} \tilde{F}_{\alpha, \beta}^{\prime}(r)=\frac{3}{R_{\beta}^{2} \operatorname{arsinh}\left(R_{\beta}\right)^{3}}>0
$$

which shows strict convexity.

We need the following basic properties of the functions $R$ and $\tilde{R}$.

Lemma 2. The following properties hold:

(1) For all $\beta \in \mathbb{R}_{+}$we have $R(\beta)=\tilde{R}(\beta, \beta)$.

(2) The functions $R$ and $\tilde{R}(\cdot, \beta)$ (with $\beta \in \mathbb{R}_{+}$fixed) are monotone increasing maps onto $\mathbb{R}_{+}$.

Proof. (1) For all $\beta, r \in \mathbb{R}_{+}$we have directly from the definitions of the functions $\tilde{F}_{\alpha, \beta}$ and $F_{\beta}$ that $\tilde{F}_{\beta, \beta}(r) \geq F_{\beta}(r)$ and $\tilde{F}_{\beta, \beta}\left(R_{\beta}\right)=F_{\beta}\left(R_{\beta}\right)$. Thus, $R_{\beta}$ does not only minimize $F_{\beta}$ but also $\tilde{F}_{\beta, \beta}$. However, the minimizer of $\tilde{F}_{\beta, \beta}$ is uniquely determined. Thus, $\tilde{R}_{\beta, \beta}=R_{\beta}$.

(2) $R(\beta)$ solves the equation $F_{\beta}^{\prime}(r)=0$. From (23) it is obvious that the function $R$ is monotone increasing. It is also obvious from (23) that $R_{\beta} \rightarrow 0$ as $\beta \rightarrow 0$ and $R_{\beta} \rightarrow \infty$ as $r \rightarrow \infty$. This shows the claim on $R$. 
By strict convexity, $\tilde{F}_{\alpha \beta}^{\prime}(r)<0$ for $r<\tilde{R}_{\alpha, \beta}$ and $\tilde{F}_{\alpha, \beta}^{\prime}(r)>0$ for $r>\tilde{R}_{\alpha, \beta}$ holding for all $\alpha, \beta \in \mathbb{R}_{+}$, holding in particular for $\alpha=\beta$. Moreover, for $\alpha>\beta$ and $r<R_{\beta}$ we have

$$
\tilde{F}_{\alpha, \beta}^{\prime}(r)=-\frac{4}{r^{5}}\left(\frac{R_{\beta}}{\operatorname{arsinh}\left(R_{\beta}\right)}\right)^{3}+\frac{1}{\alpha}<F_{\beta, \beta}^{\prime}(r)<0,
$$

i.e., $\tilde{R}_{\alpha, \beta} \geq R_{\beta}$ for $\alpha>\beta$. Away from $R_{\beta}$ we can use again the Euler equation: suppose that there is a $\alpha^{\prime} \in \mathbb{R}_{+}$such that $\tilde{R}_{\alpha^{\prime}, \beta}>R_{\beta}$. Then $\tilde{R}_{\alpha^{\prime}, \beta}$ fulfills

$$
\tilde{F}_{\alpha^{\prime}, \beta}^{\prime}\left(\tilde{R}_{\alpha^{\prime}, \beta}\right)=-\frac{1}{\tilde{R}_{\alpha^{\prime}, \beta}^{2} \operatorname{arsinh}\left(R_{\beta}\right)^{3}}+\frac{1}{\alpha^{\prime}}=0 .
$$

Thus, we have for any $\alpha^{\prime \prime}>\alpha^{\prime}$, an $r^{\prime}>\tilde{R}_{\alpha^{\prime}, \beta}>R_{\beta}$ solving the equation

$$
\tilde{F}_{\alpha^{\prime \prime}, \beta}^{\prime}(r)=-\frac{1}{r^{2} \operatorname{arsinh}\left(R_{\beta}\right)^{3}}+\frac{1}{\alpha^{\prime \prime}}=0 .
$$

Since $\tilde{R}_{\alpha^{\prime \prime}, \beta}>R_{\beta}$, we have $r^{\prime}=\tilde{R}_{\alpha^{\prime \prime}, \beta}$. Thus $\tilde{R}_{\alpha^{\prime \prime}, \beta}>\tilde{R}_{\alpha^{\prime}, \beta}$ for $\alpha^{\prime \prime}>\alpha^{\prime}>\beta$. This implies the monotony in $\alpha$ for $\alpha>\beta$. Similar arguments yield the monotony in $\alpha$ for $\alpha \leq \beta$.

Proof of Theorem 1. The condition $\nabla(F \circ p) \in L^{2}\left(\mathbb{R}^{3}\right)$ is a mere rewriting of the finiteness condition of the Weizsäcker term. Similarly $D\left[p^{3}\right]<\infty$ is equivalent to the finiteness of the electron-electron repulsion.

Next we look at the Thomas-Fermi term. Obviously the massive Thomas-Fermi term is bounded above by the massless one. It is also bounded from below by the massless Thomas-Fermi term minus the particle number (see (87)), i.e.,

$$
\mathcal{T}^{\mathrm{TF}}(p) \geq \frac{1}{4 \pi^{2}} \int_{\mathbb{R}^{3}} \mathrm{~d} x p(x)^{4}-N .
$$

Both bounds are obvious from the representation of $\mathcal{T}^{\mathrm{TF}}$ as phase space integral.

Since $p \in L^{4}\left(\mathbb{R}^{3}\right), p$ decays at infinity and therefore also $F \circ p$. Thus, we may employ the Sobolev inequality yielding $F \circ p \in L^{6}\left(\mathbb{R}^{3}\right)$ and estimate $F$ from below using (90) from the appendix. We get

$$
\begin{aligned}
\int_{\mathbb{R}^{3}} \mathrm{~d} x|\nabla(F \circ p)(x)|^{2} & \geq c_{s}\left(\int_{\mathbb{R}^{3}} \mathrm{~d} x|F \circ p(x)|^{6}\right)^{1 / 3} \\
& \geq \frac{c_{s}}{4}\left(\int_{\mathbb{R}^{3}} \mathrm{~d} x p(x)^{6} \operatorname{arsinh}(p(x))^{3}\right)^{1 / 3}=: \frac{c_{s}}{4} W(p)^{1 / 3}
\end{aligned}
$$

where $c_{s}$ is the Sobolev constant. Thus, using (28), (3), and (27) we get

$$
\mathcal{E}^{\mathrm{TFW}}(p) \geq \frac{3 c_{s} \lambda}{32 \pi^{2}} W(p)^{1 / 3}+\frac{1}{4 \pi^{2}} T(p)-N-\frac{3}{10} \gamma \alpha_{S} \int_{\mathbb{R}^{3}} \mathrm{~d} x p(x)^{5}-\frac{\alpha_{S}}{\gamma} A
$$

with $T(p):=\int_{\mathbb{R}^{3}} \mathrm{~d} x p(x)^{4}$. We pick

$$
\gamma=\sqrt{\frac{A}{\frac{3}{10} \int_{\mathbb{R}^{3}} \mathrm{~d} x p(x)^{5}}}
$$

and get

$$
\mathcal{E}^{\mathrm{TFW}}(p) \geq \frac{3 \lambda c_{s}}{32 \pi^{2}} W(p)^{1 / 3}+\frac{1}{4 \pi^{2}} T(p)-N-2 \alpha_{S} \sqrt{\frac{3 A}{10} \int_{\mathbb{R}^{3}} \mathrm{~d} x p(x)^{5}} .
$$


We pick $\beta \in \mathbb{R}_{+}$to be specified later but independently of $p$ and estimate

$$
\begin{aligned}
& \int_{\mathbb{R}^{3}} \mathrm{~d} x p(x)^{5} \\
\leq & \frac{1}{r \operatorname{arsinh}(r)^{3}} \int_{p(x)>r} \mathrm{~d} x p(x)^{6} \operatorname{arsinh}(p(x))^{3}+r \int_{p(x) \leq r} \mathrm{~d} x p(x)^{4} \\
\leq & \frac{1}{r \operatorname{arsinh}(r)^{3}} W(p)+r T(p) \\
\leq & \begin{cases}\frac{1}{r \operatorname{arsinh}\left(R_{\beta}\right)^{3}} W(p)+r T(p) & r \geq R_{\beta} \\
\frac{1}{r^{4}} W(p)\left(R_{\beta} / \operatorname{arsinh}\left(R_{\beta}\right)\right)^{3}+r T(p) & r<R_{\beta}\end{cases}
\end{aligned}
$$

where $R_{\beta}$ is the unique minimizer of the function $F_{\beta}$ defined in (21). Using (22) allows us to rewrite (34) so that we get

$$
\int_{\mathbb{R}^{3}} \mathrm{~d} x p(x)^{5} \leq W(p) \tilde{F}_{Q, \beta}(r)
$$

in the variable $r$. Here we use the abbreviation $Q:=W(p) / T(p)$. The resulting minimizer $\tilde{R}_{Q, \beta}$ exists uniquely for each $p$ and $\beta$ by Lemma 1. Inserting it into (35) gives

$$
\begin{gathered}
\int_{\mathbb{R}^{3}} \mathrm{~d} x p(x)^{5} \leq W(p) \tilde{F}_{Q, \beta}\left(\tilde{R}_{Q, \beta}\right) \\
=\left\{\begin{array}{ll}
\frac{1}{\tilde{R}_{Q, \beta} \operatorname{arsinh}\left(R_{\beta}\right)^{3}} W(p)+\tilde{R}_{Q, \beta} T(p) & Q \geq \beta \\
\frac{1}{R_{Q, \beta}^{4}} W(p)\left(R_{\beta} / \operatorname{arsinh}\left(R_{\beta}\right)\right)^{3}+R_{Q, \beta} T(p) & Q<\beta
\end{array} .\right.
\end{gathered}
$$

In the last step we used that $\tilde{R}_{\alpha, \beta}$ is monotone increasing in $\alpha$ and $\tilde{R}_{\beta, \beta}=R_{\beta}$.

The optimizer of the first line is

$$
\tilde{R}_{Q, \beta}=\sqrt{\frac{W(p)}{T(p) \operatorname{arsinh}\left(R_{\beta}\right)^{3}}} .
$$

The second line is minimized for

$$
\tilde{R}_{Q, \beta}=\sqrt[5]{\frac{4 W(p) R_{\beta}^{3}}{\operatorname{arsinh}\left(R_{\beta}\right)^{3} T(p)}} .
$$

Thus,

$$
\begin{aligned}
\int_{\mathbb{R}^{3}} \mathrm{~d} x p(x)^{5} & \leq \begin{cases}2 \sqrt{\frac{W(p) T(p)}{\operatorname{arsinh}\left(R_{\beta}\right)^{3}}} & W(p) \geq \beta T(p) \\
\frac{5}{4^{4 / 5}}\left(W(p)\left(R_{\beta} / \operatorname{arsinh}\left(R_{\beta}\right)^{3}\right)^{1 / 5} T(p)^{4 / 5}\right. & W(p)<\beta T(p)\end{cases} \\
& \leq \begin{cases}2 \sqrt{\frac{W(p) T(p)}{\operatorname{arsinh}\left(R_{\beta}\right)^{3}}} & W(p) \geq \beta T(p) \\
\frac{5}{4^{4 / 5}}\left(\beta\left(R_{\beta} / \operatorname{arsinh}\left(R_{\beta}\right)^{3}\right)^{1 / 5} T(p)\right. & W(p)<\beta T(p)\end{cases}
\end{aligned}
$$

We insert this bound in (30) and obtain

$$
\begin{aligned}
\mathcal{E}^{\mathrm{TFW}}(p) \geq & \frac{3 \lambda c_{s}}{32 \pi^{2}} W(p)^{1 / 3}+\frac{1}{4 \pi^{2}} T(p)-N \\
& -2 \alpha_{S} \begin{cases}\sqrt{\frac{3 A}{5 \sqrt{2 \operatorname{arsinh}\left(R_{\beta}\right)^{3}}}}\left(\frac{W(p)^{1 / 3}}{\frac{4}{3}}+\frac{T(p)}{4}\right) & W(p) \geq \beta T(p) \\
\sqrt{\frac{3}{2} \frac{A}{4^{4 / 5}}\left(\beta R_{\beta}^{3} / \operatorname{arsinh}\left(R_{\beta}\right)^{3}\right)^{1 / 5} T(p)} & W(p)<\beta T(p) .\end{cases}
\end{aligned}
$$

Now, we pick $\beta$ such that

$$
\min \left\{\frac{\lambda c_{s}}{8 \pi^{2}}, \frac{1}{\pi^{2}}\right\}=\sqrt{\frac{3 A}{5 \sqrt{2 \operatorname{arsinh}\left(R_{\beta}\right)^{3}}}} .
$$


This bounds the case of the first line from below by $-N$.

The second line is bounded from below - independently of $p$, since the leading power in $T(p)$ has a positive coefficient. To make this quantitative, we write $a$ for the coefficient of $T(p)$ and $b$ for the coefficient of $T(p)$ in the second case of (39). Now $a T(p)-b \sqrt{T(p)}$ is minimized for $T(p)=b^{2} /\left(4 a^{2}\right)$ implying

$$
a T(p)-b \sqrt{T(p)} \geq-b^{2} /(4 a)=: C(A)
$$

independently of $p$.

Since (40) implies that $R_{\beta}$ is increasing in $A$, we find that $C(A)$ is increasing. Moreover by the definition of $A$ in (3), we can estimate $A \leq e_{\mathrm{TF}} Z_{\infty}^{7 / 3} \cdot K$. Thus, the second case of (39) is bounded from below by $-N-C\left(e_{\mathrm{TF}} Z_{\infty}^{7 / 3} \cdot K\right)$. Writing the latter constant as $c\left(Z_{\infty}^{7 / 3} \cdot K\right)$ yields (19).

\section{Proof of the Existence of Minimizers (Theorem 21)}

Since $\mathcal{E}^{\mathrm{TFW}}$ is bounded from below on $P_{N}$, we will now address the question whether the infimum is attained. It is convenient, to regard the functional as function of the density $\rho$ instead of the Fermi momentum $p$ and similarly for other parts of the energy functional. In abuse of notation we write $\mathcal{E}^{\mathrm{TFW}}(\rho)$ instead of $\mathcal{E}^{\mathrm{TFW}}\left(\sqrt[3]{3 \pi^{2} \rho}\right)$, i.e.

$$
\mathcal{E}^{\mathrm{TFW}}(\rho)
$$

$$
\begin{aligned}
= & \frac{3 \lambda}{8 \pi^{2}} \int_{\mathbb{R}^{3}} \mathrm{~d} x\left|\nabla\left(F \circ \sqrt[3]{3 \pi^{2} \rho}\right)(x)\right|^{2}+\mathcal{T}^{\mathrm{TF}}(\rho)-\alpha_{S} \sum_{k=1}^{K} \int_{\mathbb{R}^{3}} \mathrm{~d} x \frac{Z_{k} \rho(x)}{\left|x-R_{k}\right|}+\alpha_{S} D[\rho] \\
& +\sum_{1 \leq k<l \leq K} \frac{\alpha_{S} Z_{k} Z_{l}}{\left|R_{k}-R_{l}\right|} .
\end{aligned}
$$

Theorem 4. For every $N, Z_{1}, \ldots, Z_{K} \in \mathbb{R}_{+}$and $R_{1}, \ldots, R_{K} \in \mathbb{R}^{3}$ there exists $\rho \in P_{N}$ such that

$$
\mathcal{E}^{\mathrm{TFW}}(\rho)=\inf \mathcal{E}^{\mathrm{TFW}}\left(P_{N}\right) .
$$

Since $\mathcal{E}^{\mathrm{TFW}}$ has a lower bound, there is a minimizing sequence $\rho_{j}$, such that

$$
\lim _{j \rightarrow \infty} \mathcal{E}^{\mathrm{TFW}}\left(\rho_{j}\right)=\inf \mathcal{E}^{\mathrm{TFW}}\left(P_{N}\right) .
$$

However (39) shows not only boundedness from below but also that $\mathcal{E}^{\mathrm{TFW}}\left(\rho_{j}\right) \rightarrow \infty$ as either of the norms $\left\|\rho_{j}\right\|_{L^{4 / 3}\left(\mathbb{R}^{3}\right)},\left\|F \circ \sqrt[3]{3 \pi^{2} \rho_{j}}\right\|_{D^{1}\left(\mathbb{R}^{3}\right)}$, or $\left\|\rho_{j}\right\|_{C}:=\sqrt{D[\rho]}$ tend to infinity. By the Banach-Alaoglu theorem we can pick a subsequence, such that we have weak convergence in all of these norms. We now imagine that we started already with this subsequence to avoid a new notation. We have $\chi \in D^{1}\left(\mathbb{R}^{3}\right)$, $\zeta_{2} \in L^{4 / 3}\left(\mathbb{R}^{3}\right), \zeta_{3}$ with $D\left[\zeta_{3}\right]<\infty$, and $\zeta_{4} \in L^{5 / 3}(\mathbb{R})$ such that, as $j \rightarrow \infty$,

$$
\begin{array}{rlrl}
\int_{\mathbb{R}^{3}} \mathrm{~d} x \nabla f(x) \nabla\left(F \circ \sqrt[3]{3 \pi^{2} \rho_{j}}-\chi\right)(x) \rightarrow 0 & & \forall f \in D^{1}\left(\mathbb{R}^{3}\right), \\
\int_{\mathbb{R}^{3}} \mathrm{~d} x f(x)\left(\rho_{j}-\zeta_{2}\right)(x) \rightarrow 0 & \forall f \in L^{4}\left(\mathbb{R}^{3}\right), \\
D\left(f, \rho_{j}-\zeta_{3}\right) \rightarrow 0 & \forall f \text { with } D[f]<\infty, \\
\int_{\mathbb{R}^{3}} \mathrm{~d} x f(x)\left(\rho_{j}-\zeta_{4}\right)(x) \rightarrow 0 & \forall f \in L^{5 / 2}\left(\mathbb{R}^{3}\right)
\end{array}
$$

using the abbreviation (2). The convergence (48) holds, since by (38), $\left\|\rho_{j}\right\|_{L^{5 / 3}\left(\mathbb{R}^{3}\right)}$ is also bounded. 
To prove the existence of the minimizer, we prove the lower semicontinuity of each term of $\mathcal{E}^{\mathrm{TFW}}$.

We begin with the Weizsäcker term. Since the norm on $D^{1}\left(\mathbb{R}^{3}\right)$ is lower continuous (Lieb and Loss [21, Section 8.2]), we immediately have

$$
\mathcal{T}^{\mathrm{W}}(\zeta) \leq \liminf _{j \rightarrow \infty} \mathcal{T}^{\mathrm{W}}\left(\rho_{j}\right)
$$

with $\zeta:=\left(3 \pi^{2}\right)^{-1}\left(F^{-1}(\chi)\right)^{3}$.

Next we consider the $\mathcal{T}^{\mathrm{TF}}$ which obviously is a convex functional with a derivative $d$ at $\rho$ (see also Appendix $\mathrm{A}$ )

$$
\begin{aligned}
{\left[d\left(\mathcal{T}^{\mathrm{TF}}\right)(\rho)\right]: L^{4 / 3} } & \rightarrow \mathbb{R} \\
\eta & \mapsto \int_{\mathbb{R}^{3}} \mathrm{~d} x\left(\sqrt{\left(3 \pi^{2} \rho\right)^{2 / 3}+1}-1\right) \eta(x) .
\end{aligned}
$$

Since $d\left(\mathcal{T}^{\mathrm{TF}}\right)(\rho)$ is in the dual space of $L^{4 / 3}$. Therefore, we get by convexity

$$
\liminf _{j \rightarrow \infty} \mathcal{T}^{\mathrm{TF}}\left(\rho_{j}\right) \geq \mathcal{T}^{\mathrm{TF}}\left(\zeta_{2}\right)+\liminf _{j \rightarrow \infty} d\left(\mathcal{T}^{\mathrm{TF}}\right)\left(\eta_{2}\right)\left(\rho_{j}-\zeta_{2}\right)=\mathcal{T}^{\mathrm{TF}}\left(\zeta_{2}\right) .
$$

The third term, the external potential, is actually weakly continuous at the limiting element. Because of linearity and translational invariance it suffices to show this for the Coulomb potential of a unit point charge at the origin. We decompose it into a singular and a long range part

$$
1 /|x|:=l(x)+r(x)
$$

with

$$
r(|x|):=\int_{|y|<1} \mathrm{~d} y \frac{\mu(y)}{|x-y|},
$$

where we pick some spherically symmetric charge distribution supported in the unit ball centered at the origin with $D[\mu]<\infty$. Whereas $l \in L^{5 / 2}, \operatorname{since} \operatorname{supp}(l) \subset B_{1}(0)$ and $l(x) \leq 1 /|x|$ because of Newton's theorem (see [23, Section III.9]).

Thus

$$
\lim _{j \rightarrow \infty} \int_{\mathbb{R}^{3}} \mathrm{~d} x r(x) \rho_{j}(x)=2 \lim _{j \rightarrow \infty} D\left(\mu, \rho_{j}\right)=2 D\left(\mu, \zeta_{3}\right)=\int_{\mathbb{R}^{3}} \mathrm{~d} x r(x) \zeta_{3}(x) .
$$

Since $l \in L^{5 / 2}\left(\mathbb{R}^{3}\right)$ we have

$$
\lim _{j \rightarrow \infty} \int_{\mathbb{R}^{3}} \mathrm{~d} x l(x) \rho_{j}(x)=\lim _{j \rightarrow \infty} \int_{\mathbb{R}^{3}} \mathrm{~d} x l(x) \zeta_{4}(x) .
$$

Eventually we consider the electron-electron repulsion. By the Schwarz inequality

and thus

$$
D\left(\zeta_{3}, \zeta_{3}\right)=\lim _{j \rightarrow \infty} D\left(\rho_{j}, \zeta_{3}\right) \leq \liminf _{j \rightarrow \infty} \sqrt{D\left[\rho_{j}\right] D\left[\zeta_{3}\right]}
$$

$$
D\left[\zeta_{3}\right] \leq \liminf _{j \rightarrow \infty} D\left[\rho_{j}\right] .
$$

Thus all the terms are lower continuous at the corresponding limiting points. To conclude the proof, we wish to show that $\zeta=\zeta_{2}=\zeta_{3}=\zeta_{4}$ and that the limiting point is in $P_{N}$.

Since $C_{0}^{\infty}\left(\mathbb{R}^{3}\right) \subset L^{4}\left(\mathbb{R}^{3}\right) \cap L^{5 / 2}\left(\mathbb{R}^{3}\right)$ we have for all $f \in C_{0}^{\infty}\left(\mathbb{R}^{3}\right)$ the two equalities

$$
\int_{\mathbb{R}^{3}} \mathrm{~d} x f(x) \zeta_{2}=\lim _{j \rightarrow \infty} \int_{\mathbb{R}^{3}} \mathrm{~d} x f(x) \rho_{j}(x)=\int_{\mathbb{R}^{3}} \mathrm{~d} x f(x) \zeta_{4},
$$

i.e., $\zeta_{2}=\zeta_{4}$. 
Next we take $-(4 \pi)^{-1} \Delta f$ with $f \in C_{0}^{\infty}\left(\mathbb{R}^{3}\right)$. Then

$$
\begin{aligned}
\int_{\mathbb{R}^{3}} \mathrm{~d} x f(x) \zeta_{3}(x)=D\left(-(4 \pi)^{-1} \Delta f, \zeta_{3}\right)=\lim _{j \rightarrow \infty} D\left(-(4 \pi)^{-1} \Delta f, \rho_{j}\right) \\
=\lim _{j \rightarrow \infty} \int_{\mathbb{R}^{3}} \mathrm{~d} x f(x) \rho_{j}(x)=\int_{\mathbb{R}^{3}} \mathrm{~d} x f(x) \zeta_{2}(x) .
\end{aligned}
$$

Thus $\zeta_{2}=\zeta_{3}$.

By [21, Theorem 8.7] we can also assume that $\chi_{j}:=\left.F\left(\sqrt[3]{3 \pi^{2} \rho_{j}}\right) \rightarrow \chi\right|_{A}$ converges in $L^{6}(A)$ strongly on sets $A \subset \mathbb{R}^{3}$ of finite measure. We will show now, that $\rho_{j}$ converges strongly in $L^{4 / 3}(A)$ to $\left.\zeta\right|_{A}$ and thus $\zeta=\zeta_{2}$, since $A$ is arbitrary. To do this we will use an estimate on the inverse of $F$ that follows from Lemma 3 of Appendix B.

$$
\begin{aligned}
& \left(3 \pi^{2}\right)^{4 / 3} \int_{A} \mathrm{~d} x\left|\rho_{j}(x)-\zeta(x)\right|^{4 / 3}=\int_{A} \mathrm{~d} x\left|\left(F^{-1}\left(\chi_{j}(x)\right)\right)^{3}-\left(F^{-1}(\chi(x))\right)^{3}\right|^{4 / 3} \\
& \leq \underset{\text { by }}{\leq} 8 \int_{A} \mathrm{~d} x\left|\chi_{j}(x)^{2}\left(\chi_{j}(x)+1\right)-\chi(x)^{2}(\chi(x)+1)\right|^{4 / 3} \\
& \leq 8 \int_{A} \mathrm{~d} x\left|\left(\chi_{j}(x)-\chi(x)\right)\left(\chi_{j}(x)^{2}+\chi_{j}(x) \chi(x)+\chi(x)^{2}+\chi_{j}(x)+\chi(x)\right)\right|^{4 / 3} \\
& \leq 8\left(\int_{A} \mathrm{~d} x\left|\chi_{j}(x)-\chi(x)\right|^{9 / 2}\right)^{2 / 9}\left(\int_{A} \mathrm{~d} x\left|\chi_{j}^{2}+\chi_{j} \chi+\chi^{2}+\chi_{j}+\chi\right|^{12 / 7}\right)^{7 / 9}
\end{aligned}
$$

which tends to zero, since the last factor is uniformly bounded in $j$.

Finally, $\zeta \in P_{N}$, since otherwise this would lead to immediate contradictions to $\zeta \geq 0$ or $\int_{\mathbb{R}} \mathrm{d} x \zeta(x) \leq N$.

\section{Proof of a Lower Bound on the Maximal Ionization}

Proof. We will now assume that $N \leq Z$ and - contradictory to the assumption of the theorem that the minimizer has a particle number that is strictly less than $N$, i.e., $\int_{\mathbb{R}^{3}} \mathrm{~d} x p_{N}(x)^{3} / 3 \pi^{2}<N$. If this is the case, then $p_{N}$ fulfills the Thomas-FermiWeizsäcker equation

$$
\begin{aligned}
& \frac{8 p_{N}^{2}(x)\left(\sqrt{p_{N}^{2}(x)+1}-1\right)}{F^{\prime}\left(p_{N}\right)(x)}-6 \lambda \Delta F\left(p_{N}\right)(x)-\sum_{k=1}^{K} \frac{8 \alpha_{S} Z_{k} p_{N}^{2}(x)}{\left|x-R_{k}\right| F^{\prime}\left(p_{N}\right)(x)} \\
& +\frac{8 \alpha_{S}}{3 \pi^{2}} \int_{\mathbb{R}^{3}} \mathrm{~d} y \frac{p_{N}^{2}(x) p_{N}^{3}(y)}{|x-y| F^{\prime}\left(p_{N}\right)(x)}=0 .
\end{aligned}
$$

The following is inspired by an idea of Benguria, Brézis, and Lieb 2]. We choose $\zeta_{0} \in C_{0}^{\infty}$ the same as in Benguria, Brézis, and Lieb [2]. It is a spherically symmetric function such that $\operatorname{supp}\left(\zeta_{0}\right) \subset B_{2}(0) \backslash B_{1}(0)$. Set $\zeta_{n}(x)=\zeta_{0}(x / n)$. By (156) we have,

$$
\begin{aligned}
\int_{\mathbb{R}^{3}} \mathrm{~d} x \zeta_{n}^{2}(x)\left(8 \left(\sqrt{p_{N}^{2}(x)+1}\right.\right. & -1)-\frac{6 \lambda F^{\prime}\left(p_{N}\right)(x) \Delta F\left(p_{N}\right)(x)}{p_{N}^{2}(x)} \\
& \left.-\sum_{k=1}^{K} \frac{8 \alpha_{S} Z_{k}}{\left|x-R_{k}\right|}+\frac{8 \alpha_{S}}{3 \pi^{2}} \int_{\mathbb{R}^{3}} \mathrm{~d} y \frac{p_{N}^{3}(y)}{|x-y|}\right)=0 .
\end{aligned}
$$


Integrating by parts and using the Schwarz inequality, we have

(58)

$$
\begin{aligned}
& -\int_{\mathbb{R}^{3}} \mathrm{~d} x \zeta_{n}^{2} \frac{F^{\prime}\left(p_{N}\right) \Delta F\left(p_{N}\right)}{p_{N}^{2}} \\
= & \int_{\mathbb{R}^{3}} \mathrm{~d} x\left(2 \zeta_{n} \nabla \zeta_{n} \frac{F^{\prime}\left(p_{N}\right)}{p_{N}^{2}}+\zeta_{n}^{2}\left(\frac{F^{\prime}\left(p_{N}\right)}{p_{N}^{2}}\right)^{\prime} \nabla p_{N}\right) F^{\prime}\left(p_{N}\right) \nabla p_{N} \\
\leq & \frac{1}{\epsilon} \int_{\mathbb{R}^{3}} \mathrm{~d} x\left|\nabla \zeta_{n}\right|^{2}+\int_{\mathbb{R}^{3}} \mathrm{~d} x\left(\epsilon\left(\frac{F^{\prime 2}\left(p_{N}\right)}{p_{N}^{2}}\right)^{2}+\left(\frac{F^{\prime}\left(p_{N}\right)}{p_{N}^{2}}\right)^{\prime} F^{\prime}\left(p_{N}\right)\right) \zeta_{n}^{2}\left|\nabla p_{N}\right|^{2} .
\end{aligned}
$$

Using the definition of $F\left(p_{N}\right)$, we get

$$
\left(\frac{F^{\prime}\left(p_{N}\right)}{p_{N}^{2}}\right)^{\prime}=-\frac{\left(2 p_{N}^{2}+3\right) \sqrt{p_{N}^{2}+1}+4\left(2 p_{N}^{2}+1\right) p_{N} \operatorname{arsinh}\left(p_{N}\right)}{2 p_{N}^{\frac{5}{2}}\left(p_{N}^{2}+1\right)^{\frac{3}{2}} \sqrt{\sqrt{p_{N}^{2}+1}+2 p_{N} \operatorname{arsinh}\left(p_{N}\right)}}<0
$$

Define

$$
g(s):=-\frac{\left(\frac{F^{\prime}(s)}{s^{2}}\right)^{\prime} F^{\prime}(s)}{\left(\frac{F^{\prime 2}(s)}{s^{2}}\right)^{2}}, \quad g\left(p_{N}\right)>0 .
$$

We easily get $g\left(0_{+}\right)=3 / 2$ and $\lim _{s \rightarrow \infty} g(s)=\infty$. So $c_{g}:=\min _{s \geq 0} g(s)>0$. Choose $\epsilon=c_{g}$. Then

$$
-\int_{\mathbb{R}^{3}} \mathrm{~d} x \zeta_{n}^{2} \frac{F^{\prime}\left(p_{N}\right) \Delta F\left(p_{N}\right)}{p_{N}^{2}} \leq \frac{1}{c_{g}} \int_{\mathbb{R}^{3}} \mathrm{~d} x\left|\nabla \zeta_{n}\right|^{2} \leq C n .
$$

Next, we compute

$$
\int_{\mathbb{R}^{3}} \mathrm{~d} x \zeta_{n}^{2}\left(\sqrt{p_{N}^{2}+1}-1\right)<\int_{\mathbb{R}^{3}} \mathrm{~d} x \zeta_{n}^{2} p_{N} \leq \epsilon_{n} n^{2}
$$

where $\epsilon_{n} \rightarrow 0$ as $n \rightarrow \infty$. The last inequality is proved by Benguria, Brézis, and Lieb 2]. About the external potential term, since $\zeta_{n}(x)=0$ for $|x|<n$, we have

$$
\begin{aligned}
& \int_{\mathbb{R}^{3}} \mathrm{~d} x \zeta_{n}^{2}(x) \sum_{k=1}^{K} \frac{8 \alpha_{S} Z_{k}}{\left|x-R_{k}\right|} \\
= & \int_{\mathbb{R}^{3}} \mathrm{~d} x \zeta_{n}^{2}(x) \sum_{k=1}^{K} 8 \alpha_{S} Z_{k}\left(\frac{1}{|x|}+\frac{|x|-\left|x-R_{k}\right|}{|x|\left|x-R_{k}\right|}\right) \\
\geq & \int_{\mathbb{R}^{3}} \mathrm{~d} x \zeta_{n}^{2}(x) \sum_{k=1}^{K} 8 \alpha_{S} Z_{k}\left(\frac{1}{|x|}-\frac{\max \left\{\left|R_{k}\right|\right\}}{|x|\left(n-\max \left\{\left|R_{k}\right|\right\}\right)}\right) \\
\geq & \left(1-\frac{c}{n}\right) 8 \alpha_{S} \sum_{k=1}^{K} Z_{k} \int_{\mathbb{R}^{3}} \mathrm{~d} x \frac{\zeta_{n}^{2}(x)}{|x|}
\end{aligned}
$$


for large $n$. We now address the remaining term: since $\zeta_{n}$ is spherically symmetric, by a result of Lieb and Simon [22, Eq. (35)] we have

$$
\begin{aligned}
& \int_{\mathbb{R}^{3}} \mathrm{~d} x \zeta_{n}^{2}(x) \frac{8 \alpha_{S}}{3 \pi^{2}} \int_{\mathbb{R}^{3}} \mathrm{~d} y \frac{p_{N}^{3}(y)}{|x-y|} \\
= & \int_{\mathbb{R}^{3}} \mathrm{~d} x \zeta_{n}^{2}(x)\left[\frac{8 \alpha_{S}}{3 \pi^{2}} \int_{\mathbb{R}^{3}} \mathrm{~d} y \frac{p_{N}^{3}(y)}{|x-y|}\right] \\
= & \int_{\mathbb{R}^{3}} \mathrm{~d} x \zeta_{n}^{2}(x) \frac{8 \alpha_{S}}{3 \pi^{2}} \int_{\mathbb{R}^{3}} \mathrm{~d} y \frac{p_{N}^{3}(y)}{\max (|x|,|y|)} \\
\leq & 8 \alpha_{S} N \int_{\mathbb{R}^{3}} \mathrm{~d} x \frac{\zeta_{n}^{2}(x)}{|x|},
\end{aligned}
$$

where $[\varphi]$ denotes the spherical average of $\varphi$, i.e.,

$$
[\varphi](x)=\frac{1}{4 \pi} \int_{\mathbb{S}^{2}} \mathrm{~d} \Omega \varphi(|x| \Omega) .
$$

Thus, for large $n$, we find

$$
\int_{\mathbb{R}^{3}} \mathrm{~d} x \zeta_{n}^{2}(x)\left(-\sum_{k=1}^{K} \frac{8 \alpha_{S} Z_{k}}{\left|x-R_{k}\right|}+\frac{8 \alpha_{S}}{3 \pi^{2}} \int_{\mathbb{R}^{3}} \mathrm{~d} y \frac{p_{N}^{3}(y)}{|x-y|}\right) \leq c(N-Z) n^{2}+c n .
$$

Combining (57), (61), (62), and (65), we find

$$
\epsilon_{n} n^{2}+C n+c(N-Z) n^{2} \geq 0 .
$$

As $n \rightarrow \infty$, we have that $Z \leq N$ which contradicts the assumption that $N<Z$.

\section{Proof of the Upper Bound on the Maximal Ionization}

Proof of $N \leq$ const $Z$. It will be convenient to express the TFW functional in terms of

$$
\chi:=F \circ p
$$

which is guided by the idea to make the dominating term in the energy simple. Obviously $F$ is strictly monotone and $F\left(\mathbb{R}_{+}\right)=\mathbb{R}_{+}$. We get

(68) $\quad \tilde{\mathcal{E}}^{\mathrm{TFW}}(\chi):=\mathcal{E}^{\mathrm{TFW}}\left(F^{-1} \circ \chi\right)$

$$
\begin{gathered}
=\frac{3 \lambda}{8 \pi^{2}} \int_{\mathbb{R}^{3}} \mathrm{~d} x|\nabla \chi(x)|^{2}+\frac{1}{8 \pi^{2}} \int_{\mathbb{R}^{3}} \mathrm{~d} x t^{\mathrm{TF}} \circ F^{-1} \circ \chi(x) \\
-\sum_{k=1}^{K} \frac{\alpha_{S} Z_{k}}{3 \pi^{2}} \int_{\mathbb{R}^{3}} \mathrm{~d} x \frac{\left(F^{-1}(\chi(x))\right)^{3}}{\left|x-R_{k}\right|}+\frac{\alpha_{S}}{9 \pi^{4}} D\left[\left(F^{-1} \circ \chi\right)^{3}\right]+\sum_{1 \leq k<l \leq K} \frac{\alpha_{S} Z_{k} Z_{l}}{\left|R_{k}-R_{l}\right|} .
\end{gathered}
$$

Suppose that $\chi$ minimizes the TFW functional on $F\left(P_{N}\right)$. Then it satisfies

$$
\begin{aligned}
& \text { (69) } \quad-\frac{3 \lambda}{4 \pi^{2}} \Delta \chi+\frac{1}{8 \pi^{2}} T^{\prime}\left(F^{-1}(\chi)\right)\left(F^{-1}\right)^{\prime}(\chi) \\
& -\sum_{k=1}^{K} \frac{Z_{k} \alpha_{S}}{\pi^{2}} \frac{\left(F^{-1}\right)^{\prime}(\chi)\left(F^{-1}(\chi)\right)^{2}}{\left|x-R_{k}\right|}+\frac{\alpha_{S}}{3 \pi^{4}}\left(F^{-1}\right)^{\prime}(\chi)\left(F^{-1}(\chi)\right)^{2} \int_{\mathbb{R}^{3}} \mathrm{~d} y \frac{\left(F^{-1}(\chi(y))\right)^{3}}{|x-y|}=0 .
\end{aligned}
$$

Following the spirit of Lieb [19], we multiply by $\chi / \phi(x)$ and integrate. The function $\phi$ is defined as

$$
\phi(x):=\sum_{k=1}^{K} \frac{\kappa_{k}}{\left|x-R_{k}\right|}
$$


The coefficients $\kappa_{k}>0$ will be given later. Due to our transform the Weizsäcker term becomes easy. We can follow Lieb's argument [19, Eq. (3.17)] step by step to see that it is positive.

Since $F$ is the antiderivative of a positive expression, $F^{\prime}$ is positive and thus also the derivative of $F^{-1}$. Therefore

$$
\int_{\mathbb{R}^{3}} \mathrm{~d} x \frac{\chi(x)}{\phi(x)} t^{\mathrm{TF}^{\prime}}\left(F^{-1}(\chi)\right)\left(F^{-1}\right)^{\prime}(\chi(x))>0
$$

when $\chi$ does not vanish almost everywhere.

Thus we have the inequality

$$
\begin{aligned}
-\sum_{k=1}^{K} Z_{k} \int_{\mathbb{R}^{3}} \mathrm{~d} x \frac{\chi(x)}{\phi(x)\left|x-R_{k}\right|}\left(F^{-1}\right)^{\prime}(\chi(x))\left(F^{-1}(\chi(x))\right)^{2} \\
+\frac{1}{3 \pi^{2}} \int_{\mathbb{R}^{3}} \mathrm{~d} x \int_{\mathbb{R}^{3}} \mathrm{~d} y \frac{\chi(x)\left(\left(F^{-1}\right)^{\prime}(\psi(x))\right)^{2}\left(F^{-1}(\chi(y))\right)^{3}}{\phi(x)|x-y|}<0
\end{aligned}
$$

where we assumed that $\chi$ is not vanishing almost everywhere, which we may, since otherwise the claim is trivial. Rewriting this in $p$ it becomes

$$
\begin{aligned}
-3 \pi^{2} \sum_{k=1}^{K} Z_{k} \int_{\mathbb{R}^{3}} \mathrm{~d} x \frac{F(p(x)) / F^{\prime}(p(x)) p(x)^{2}}{\phi(x)\left|x-R_{k}\right|} \\
\quad+\int_{\mathbb{R}^{3}} \mathrm{~d} x \int_{\mathbb{R}^{3}} \mathrm{~d} y \frac{F(p(x)) / F^{\prime}(p(x)) p(x)^{2} p(y)^{3}}{\phi(x)|x-y|}<0 .
\end{aligned}
$$

We now analyze the minimum $a$ and maximum $b$ of the function $H$ (see (15) and (16)). That $b=1$ is easily seen from the fact, that $f^{\prime}(t)>0$ for all $t>0$ which implies that $F(t):=\int_{0}^{t} \mathrm{~d} s f(s) \leq f(t) t$. That $a>0$ follows from the bounds given in Lemma 3 of Appendix B. Then (72) is equivalent to

$$
-3 \pi^{2} \sum_{k=1}^{K} Z_{k} \int_{\mathbb{R}^{3}} \mathrm{~d} x \frac{H(x) p(x)^{3}}{\phi(x)\left|x-R_{k}\right|}+\int_{\mathbb{R}^{3}} \mathrm{~d} x \int_{\mathbb{R}^{3}} \mathrm{~d} y \frac{H(x) p(x)^{3} p(y)^{3}}{\phi(x)|x-y|}<0 .
$$

This and symmetrizing the second integrand in $x$ allows us to turn (72) into two new inequalities

$$
\begin{aligned}
& \frac{a}{2} \int_{\mathbb{R}^{3}} \mathrm{~d} x \int_{\mathbb{R}^{3}} \mathrm{~d} y \frac{\phi(x)^{-1}+\phi(y)^{-1}}{|x-y|} p(x)^{3} p(y)^{3} \\
< & 3 \pi^{2} \sum_{k=1}^{K} Z_{k} \int_{\mathbb{R}^{3}} \mathrm{~d} x g_{k}(x) H(x) p(x)^{3}
\end{aligned}
$$

and

$$
\begin{aligned}
& \frac{1}{2} \int_{\mathbb{R}^{3}} \mathrm{~d} x \int_{\mathbb{R}^{3}} \mathrm{~d} y \frac{\phi(x)^{-1}+\phi(y)^{-1}}{|x-y|} H(x) p(x)^{3} H(y) p(y)^{3} \\
< & 3 \pi^{2} \sum_{k=1}^{K} Z_{k} \int_{\mathbb{R}^{3}} \mathrm{~d} x g_{k}(x) H(x) p(x)^{3},
\end{aligned}
$$

where $g_{k}(x):=\left(\phi(x)\left|x-R_{k}\right|\right)^{-1}$. Following an idea of Baumgartner [1] and using the triangular inequality, we have

$$
\frac{\phi(x)^{-1}+\phi(y)^{-1}}{|x-y|}=\sum_{k=1}^{K} \kappa_{k} \frac{\left|x-R_{k}\right|+\left|y-R_{k}\right|}{|x-y|} g_{k}(x) g_{k}(y) \geq \sum_{k=1}^{K} \kappa_{k} g_{k}(x) g_{k}(y) .
$$


Therefore, applying (76) on the left side of (74) and (75) yields

$$
\begin{aligned}
& \text { (77) } \quad \frac{a}{2} \sum_{k=1}^{K} \kappa_{k}\left(\int_{\mathbb{R}^{3}} \mathrm{~d} x g_{k}(x) p(x)^{3}\right)^{2}<3 \pi^{2} \sum_{k=1}^{K} Z_{k} \int_{\mathbb{R}^{3}} \mathrm{~d} x g_{k}(x) H(x) p(x)^{3}, \\
& \text { (78) } \frac{1}{2} \sum_{k=1}^{K} \kappa_{k}\left(\int_{\mathbb{R}^{3}} \mathrm{~d} x g_{k}(x) H(x) p(x)^{3}\right)^{2}<3 \pi^{2} \sum_{k=1}^{K} Z_{k} \int_{\mathbb{R}^{3}} \mathrm{~d} x g_{k}(x) H(x) p(x)^{3} .
\end{aligned}
$$

Adding the two inequalities, we have

$$
\begin{aligned}
& 6 \pi^{2} \sum_{k=1}^{K} Z_{k} \int_{\mathbb{R}^{3}} \mathrm{~d} x g_{k}(x) H(x) p(x)^{3} \\
& >\sum_{k=1}^{K} \kappa_{k}\left(\frac{a}{2}\left(\int_{\mathbb{R}^{3}} \mathrm{~d} x g_{k}(x) p(x)^{3}\right)^{2}+\frac{1}{2}\left(\int_{\mathbb{R}^{3}} \mathrm{~d} x g_{k}(x) H(x) p(x)^{3}\right)^{2}\right) \\
& \geq \sum_{k=1}^{K} \kappa_{k} \sqrt{a} \int_{\mathbb{R}^{3}} \mathrm{~d} x g_{k}(x) p(x)^{3} \int_{\mathbb{R}^{3}} \mathrm{~d} x g_{k}(x) H(x) p(x)^{3} .
\end{aligned}
$$

This is equivalent to

$$
\sum_{k=1}^{K} \int_{\mathbb{R}^{3}} \mathrm{~d} x g_{k}(x) H(x) p(x)^{3}\left(6 \pi^{2} Z_{k}-\sqrt{a} \kappa_{k} \int_{\mathbb{R}^{3}} \mathrm{~d} x g_{k}(x) p(x)^{3}\right)>0 .
$$

Following Lieb's [19] setting, let

$$
\begin{aligned}
\delta_{k} & :=\frac{\kappa_{k}}{3 \pi^{2} N} \int_{\mathbb{R}^{3}} \mathrm{~d} x g_{k}(x) p(x)^{3}, \\
\nu_{k} & :=Z_{k} / Z .
\end{aligned}
$$

Note that

$$
\sum_{k=1}^{K} \delta_{k}=\sum_{k=1}^{K} \nu_{k}=1
$$

It is proved by Lieb [19, Appendix B] that we can choose $\kappa_{k}$ such that

$$
\delta_{k}=\nu_{k}, k=1, \ldots, K \text {. }
$$

Then the left hand side of (80) becomes

$$
3 \pi^{2}(2 Z-\sqrt{a} N) \sum_{k=1}^{K} \int_{\mathbb{R}^{3}} \mathrm{~d} x g_{k}(x) H(x) p(x)^{3} \delta_{k}>0 .
$$

The sum is positive, so this yields

$$
N<\frac{2}{\sqrt{a}} Z
$$

or, numerically $N<2.557211758 Z$. Recall that by definition $a:=\inf H\left(\mathbb{R}_{+}\right)$(see (15) and the line below) and thus depends only on $f$.

\section{Appendix A. On the semiclassical nature of the Thomas-Fermi term}

Note that the Thomas-Fermi $\mathcal{T}^{\mathrm{TF}}$ can be rewritten to emphasize its semiclassical nature

$$
\mathcal{T}^{\mathrm{TF}}[p]=2 \int_{\mathbb{R}^{3}} \mathrm{~d} x \int_{|\xi|<p(x)} \mathrm{d} \xi \frac{\mathrm{d} \xi}{(2 \pi)^{3}}\left(\sqrt{|\xi|^{2}+1}-1\right)
$$

Writing it this way is not only mere curiosity but also shows the convexity of $\mathcal{T}^{\mathrm{TF}}$ and the bound

(87) $\quad \frac{1}{4 \pi^{2}} \int_{\mathbb{R}^{3}} \mathrm{~d} x \rho(x)^{4 / 3} \geq \mathcal{T}^{\mathrm{TF}}\left(\sqrt[3]{3 \pi^{2} \rho}\right) \geq \frac{1}{4 \pi^{2}} \int_{\mathbb{R}^{3}} \mathrm{~d} x \rho(x)^{4 / 3}-\int_{\mathbb{R}^{3}} \mathrm{~d} x \rho(x)$. 
This also allows to read off its derivative:

$$
\left(d \mathcal{T}^{\mathrm{TF}}\right)(p)(\eta)=\frac{1}{\pi^{2}} \int_{\mathbb{R}^{3}} \mathrm{~d} x p(x)^{2}\left(\sqrt{p(x)^{2}+1}-1\right) \eta(x)
$$

with $\eta \in L^{4}\left(\mathbb{R}^{3}\right)$. Obviously, $\left(d \mathcal{T}^{\mathrm{TF}}\right)(p)$ can be identified with an element in $L^{4 / 3}\left(\mathbb{R}^{3}\right)$.

Writing the TF functional in $\rho$ yields

$$
\left(d \mathcal{T}^{\mathrm{TF}} \circ \sqrt[3]{3 \pi^{2}} \cdot\right)(\rho)(\eta)=\int_{\mathbb{R}^{3}} \mathrm{~d} x\left(\sqrt{\left(3 \pi^{2} \rho(x)\right)^{2 / 3}+1}-1\right) \eta(x) .
$$

for $\eta \in L^{4 / 3}\left(\mathbb{R}^{3}\right)$. Obviously this derivative can be identified with an element in $L^{4}\left(\mathbb{R}^{3}\right)$.

\section{Appendix B. Bound on the function $F$, its Derivative, And the} THOMAS-FERMI ENERGY

Lemma 3. For all $s, t>0$

$$
\begin{aligned}
F(s) & >\tilde{F}(s):=s \sqrt{\operatorname{arsinh}(s)} / 2, \\
f(s) & >\tilde{f}(s):=\sqrt{\operatorname{arsinh}(s)}, \\
t^{\mathrm{TF}}(s) & >t^{\tilde{\mathrm{TF}}}(s):=\frac{s^{4}}{1+1 /\left(\frac{4}{5} s\right)}, \\
\tilde{F}(s) & >G(s):=\frac{s^{3 / 2}}{2 \sqrt{1+s}}, \\
G^{-1}(t) & <2 t^{2 / 3} \sqrt[3]{t+1} .
\end{aligned}
$$

Moreover, $\tilde{f}$ is monotone increasing and concave.

Proof. The monotony and concavity of $\tilde{f}(s)$ follows immediately by taking the derivatives

$$
\frac{\mathrm{d}}{\mathrm{d} s} \tilde{f}(s)=\frac{1}{2 \sqrt{\left(s^{2}+1\right) \operatorname{arsinh}(s)}}>0
$$

and

$$
\frac{\mathrm{d}^{2}}{\mathrm{~d} s^{2}} \tilde{f}(s)=-\frac{s}{2 \sqrt{\left(s^{2}+1\right)^{3} \operatorname{arsinh}(s)}}-\frac{1}{4\left(s^{2}+1\right) \operatorname{arsinh}(s)^{3 / 2}}<0 .
$$

The inequality (91) is equivalent to

$$
\frac{s}{\sqrt{1+s^{2}}}+\frac{2 s^{2}}{1+s^{2}} \operatorname{arsinh}(s)>\operatorname{arsinh}(s)
$$

or

$$
s \sqrt{1+s^{2}}>\left(1-s^{2}\right) \operatorname{arsinh}(s) .
$$

This, however, is clear, since $s>\operatorname{arsinh}(s)$.

The claim (90) follows from (91) and the concavity of $\tilde{f}(s)$. We have

$$
\begin{aligned}
F(t)=\int_{0}^{t} \mathrm{~d} s f(s)>\int_{0}^{t} \mathrm{~d} s \tilde{f}(s)= & \int_{0}^{t / 2} \mathrm{~d} s(\tilde{f}(s)+\tilde{f}(t-s)) \\
& >\int_{0}^{t / 2} \mathrm{~d} s(\tilde{f}(0)+\tilde{f}(t))=t \sqrt{\operatorname{arsinh}(t)} / 2 .
\end{aligned}
$$


Next we treat (92). To this end we remark that $t^{\mathrm{TF}}(0)=t^{\tilde{\mathrm{TF}}}(0)=0$. Moreover, the derivative of the difference is

$$
\begin{aligned}
\frac{\mathrm{d}}{\mathrm{d} s}\left(t^{\mathrm{TF}}(s)-t^{\tilde{\mathrm{T}} \mathrm{F}}(s)\right) & \\
= & \frac{128\left(\left(p+\frac{5}{4}\right)^{2}\left(p^{2}+1\right)-\left(\frac{1}{2} p^{3}+\frac{57}{32} p^{2}+\frac{5}{2} p+\frac{25}{16}\right) \sqrt{p^{2}+1}\right) p^{2}}{(4 p+5)^{2} \sqrt{p^{2}+1}}>0,
\end{aligned}
$$

which proves the result.

The inequality (93) is equivalent to the fact $\operatorname{arsinh}(s)>\frac{s}{1+s}$. Using the monotony of $G(s)$ and $G\left(2 t^{2 / 3} \sqrt[3]{t+1}\right)=\frac{2^{3 / 2} t(t+1)^{1 / 2}}{2 \sqrt{1+2 t^{2 / 3} \sqrt[3]{t+1}}}>t=G\left(G^{-1}(t)\right)$, the inequality (94) follows.

\section{AcKnowledgments}

H.C. acknowledges support by the China Scholarship Council through the LMUCSC Scholarship Program. H.S. acknowledges partial support by the Deutsche Forschungsgemeinschaft, grant SI 348/15-1 and EXC-2111-390814868. Both authors acknowledge support of the Institute for Mathematical Sciences of Singapore University through their program "Density Functionals for Many-Particle Systems: Mathematical Theory and Physical Applications of Effective Equations".

\section{REFERENCES}

[1] B. Baumgartner. On the degree of ionization in the TFW theory. Letters in Mathematical Physics, 7(5):439-441, Sep 1983.

[2] Rafael Benguria, Haïm Brézis, and Elliott H. Lieb. The Thomas-Fermi-von Weizsäcker theory of atoms and molecules. Comm. Math. Phys., 79(2):167-180, 1981.

[3] Rafael Benguria, Stefan Hoops, and Heinz Siedentop. Bounds on the excess charge and the ionization energy for the Hellmann-Weizsäcker model. Annales de l'Institut Henri Poincaré, 57(1):47-65, 1992.

[4] Rafael Benguria and Elliott H. Lieb. The most negative ion in the Thomas-Fermi-von Weizsäcker theory of atoms and molecules. J. Phys. B., 18:1045-1059, 1985.

[5] Rafael D Benguria, Michael Loss, and Heinz Siedentop. Stability of atoms and molecules in an ultrarelativistic Thomas-Fermi-Weizsäcker model. Journal of Mathematical Physics, 49(1):012302, 2008.

[6] Hongshuo Chen. On the Excess Charge Problem in Relativistic Quantum Mechanics. PhD thesis, Ludwig-Maximilians-Universität München, July 2019.

[7] E. Engel and R. M. Dreizler. Field-theoretical approach to a relativistic Thomas-Fermi-DiracWeizsäcker model. Phys. Rev. A, 35:3607-3618, May 1987.

[8] E. Engel and R. M. Dreizler. Solution of the relativistic Thomas-Fermi-Dirac-Weizsäcker model for the case of neutral atoms and positive ions. Phys. Rev. A, 38:3909-3917, Oct 1988.

[9] E. Fermi. Un metodo statistico per la determinazione di alcune proprietá dell'atomo. Atti della Reale Accademia Nazionale dei Lincei, Rendiconti, Classe di Scienze Fisiche, Matematiche e Naturali, 6(12):602-607, 1927.

[10] E. Fermi. Eine statistische Methode zur Bestimmung einiger Eigenschaften des Atoms und ihre Anwendung auf die Theorie des periodischen Systems der Elemente. Z. Phys., 48:73-79, 1928.

[11] Rupert L. Frank, Konstantin Merz, Heinz Siedentop, and Barry Simon. Proof of the strong Scott conjecture for Chandrasekhar atoms. Pure and Applied Functional Analysis, preprint arXiv:1907.04894, In press, 2019.

[12] Rupert L. Frank, Heinz Siedentop, and Simone Warzel. The ground state energy of heavy atoms: Relativistic lowering of the leading energy correction. Comm. Math. Phys., 278(2):549-566, 2008.

[13] Rupert L. Frank, Heinz Siedentop, and Simone Warzel. The energy of heavy atoms according to Brown and Ravenhall: the Scott correction. Doc. Math., 14:463-516, 2009.

[14] P. Gombás. Die statistische Theorie des Atoms und ihre Anwendungen. Springer-Verlag, Wien, 1 edition, 1949.

[15] Michael Handrek and Heinz Siedentop. On the maximal excess charge of the ChandrasekharCoulomb Hamiltonian in two dimension. Lett. Math. Phys., 103(8):843-849, 2013. 
[16] C. H. Hodges. Quantum corrections to the Thomas-Fermi approximation - the Kirzhnits method. Canadian Journal of Physics, 51(13):1428-1437, 1973.

[17] D. A. Kirzhnits. Quantum corrections to the Thomas-Fermi equation. Soviet Physics JETP, 5(1):64-71, August 1957.

[18] W. Lenz. Über die Anwendbarkeit der statistischen Methode auf Ionengitter. Z. Phys., 77:713-721, 1932.

[19] Elliott H. Lieb. Bound on the maximum negative ionization of atoms and molecules. Phys. Rev. A, 29(6):3018-3028, June 1984.

[20] Elliott H. Lieb and David A. Liberman. Numerical calculation of the Thomas-Fermi-von Weizsäcker function for an infinite atom without electron repulsion. Technical Report LA9186-MS, Los Alamos National Laboratory, Los Alamos, New Mexico, April 1982.

[21] Elliott H. Lieb and Michael Loss. Analysis. Number 14 in Graduate Studies in Mathematics. American Mathematical Society, Providence, 1 edition, 1996.

[22] Elliott H. Lieb and Barry Simon. The Thomas-Fermi theory of atoms, molecules and solids. Advances in Math., 23(1):22-116, 1977.

[23] Barry Simon. Functional Integration and Quantum Physics. Academic Press Inc. [Harcourt Brace Jovanovich Publishers], New York, 1979.

[24] Jan Philip Solovej, Thomas Østergaard Sørensen, and Wolfgang L. Spitzer. The relativistic Scott correction for atoms and molecules. Commun. Pure Appl. Math., 63:39-118, January 2010.

[25] Edward Teller. On the stability of molecules in the Thomas-Fermi theory. Rev. Mod. Phys., 34(4):627-631, October 1962.

[26] L. H. Thomas. The calculation of atomic fields. Proc. Camb. Phil. Soc., 23:542-548, 1927.

[27] Katsumi Yonei and Yasuo Tomishima. On the Weizsäcker correction to the Thomas-Fermi theory of the atom. Journal of the Physical Society of Japan, 20(6):1051-1057, 1965. 